\title{
The Lethal Interaction Following Plasmodial Fusion between Two Strains of the Myxomycete Physarum polycephalum
}

\author{
By M. J. CARLILE \\ Department of Biochemistry, Imperial College of Science and Technology, \\ London, S.W. 7
}

(Accepted for publication I I March 1972)

\begin{abstract}
SUMMARY
A lethal reaction followed fusion between plasmodia of two strains ( 15 and 29) of the Myxomycete Physarum polycephalum on a solid medium. Dead areas were seen about $5 \mathrm{~h}$ after fusion and increased in size rapidly. Strain 29 was often wholly destroyed but strain 15 commonly survived and subsequently reinvaded the dead areas. Large plasmodia of 29 were destroyed by fusion with small plasmodia of 15 , but large plasmodia of 15 survived fusion with small plasmodia of 29 . The lethal reaction did not normally occur under conditions of suboptimal nutrition, but disappearance of strain 29 still took place. It is suggested that protoplasm of strain 15 can eliminate nuclei of strain 29 , but that strain 29 has little or no ability to tolerate or eliminate nuclei of strain 15 .
\end{abstract}

\section{INTRODUCTION}

Vegetative (i.e. non-sexual) fusion between genetically unlike cells has great potentialities both as a research method in cell biology and as a technique in applied genetics. Such fusions have been achieved with cells of animals (Harris, 1970), plants (Power, Cummins \& Cocking, 1970) amoebae (Jeon, Lorch \& Danielli, I970), algae (Richter, I962) and fungi, in which group heterokaryosis is extensively utilized in genetical research (Fincham \& Day, I97I). The heterokaryons that result from cell fusion may be unstable or may give rise to a cell of inferior phenotype (Caten \& Jinks, I966). Alternatively, cell fusion may result in visible damage or be lethal, as in Neurospora crassa (Williams \& Wilson, 1966) and Thanatephorus cucumeris (Flentje \& Stretton, 1969). An understanding of these adverse effects would be of theoretical and practical interest, but, since cell fusion studies normally require sophisticated techniques, and the adverse reactions commonly involve microscopic amounts of protoplasm, progress has been slow.

In Physarum polycephalum Schweinitz, contact between two genetically identical plasmodia is followed by their fusion to form a single plasmodium. If plasmodia of different strains meet, they may or may not fuse with each other, their ability to do so being genetically determined (Carlile \& Dee, I967). One locus $(f)$ controlling fusion has been studied in detail (Poulter \& Dee, I968), but other loci can influence fusion (Poulter \& Dee, I968; Collins \& Haskins, 1970; Wheals, 1970). If fusion between plasmodia of different strains does occur, an extensive lethal reaction may result, destroying much of one or both plasmodia (Carlile \& Dee, 1967). This effect, which is visible to the naked eye, has now been studied in greater detail with two strains which consistently give an intense reaction. 


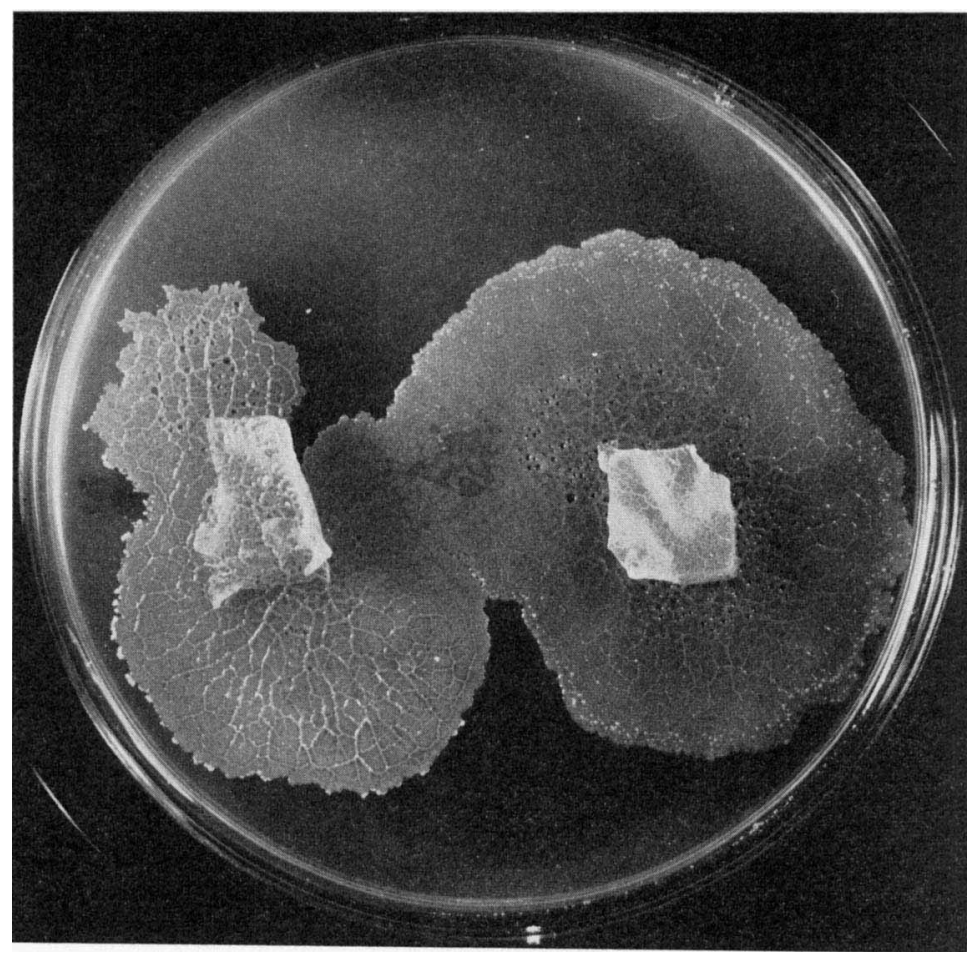

Fig. I. Lethal interaction between plasmodial strains I5 (right) and 29 of the Myxomycete Physarum polycephalum on semidefined agar medium in a $9 \mathrm{~cm}$ diameter Petri dish. Five $\mathrm{h}$ has elapsed since fusion of plasmodia and less than 5 min since the appearance of dead areas (roughly midway between the two blocks of agar used in inoculation) visible to the naked eye.

\section{METHODS}

Physarum polycephalum plasmodia, strains 15 and 29 (full designations $i+A 7015$ and $i+A$ 7029) were maintained on liquid and agar media containing $(\%, \mathrm{w} / \mathrm{v}):$ glucose, $\mathrm{r} \cdot 0$; bacteriological peptone (Oxoid), I.0; citric acid. $\mathrm{H}_{2} \mathrm{O}, 0.354 ; \mathrm{KH}_{2} \mathrm{PO}_{4}, 0 \cdot 2 ; \mathrm{CaCl}_{2} \cdot 6 \mathrm{H}_{2} \mathrm{O}$, $0.09 ; \mathrm{MgSO}_{4} \cdot 7 \mathrm{H}_{2} \mathrm{O}$, 0.06; $\mathrm{Na}_{2}$ EDTA, 0.022; $\mathrm{FeCl}_{2} \cdot{ }_{4} \mathrm{H}_{2} \mathrm{O}, 0.006 ; \mathrm{ZnSO}_{4} \cdot 7 \mathrm{H}_{2} \mathrm{O}, 0.003$; thiamin hydrochloride, 0.004 ; biotin, 0.0005 ; haem, 0.0005 ; distilled water; $\mathrm{pH}$ adjusted to 4.6. Details of the preparation and sterilization of this medium and of culture methods are given elsewhere (Carlile, 1971). Strains 15 and 29 were produced by mating amoeba of strains $A 7015$ and $A 7029$, both obtained from spores produced by plasmodial strain $A 7+i$, with amoebae of strain $i$ (Poulter \& Dee, 1968). Strains 15 and 29 are identical at their fusion loci, both having the constitution $f_{2} f_{2}$, but differ morphologically. They can be distinguished from each other if grown from equal sized inocula under identical conditions, plasmodia of 15 being paler yellow with smaller major veins and commonly a more irregular outline than plasmodia of 29 of comparable age. Experiments were carried out at $24{ }^{\circ} \mathrm{C}$ and aseptic procedures employed throughout. The criterion for recording the occurrence of a lethal reaction was the presence of dead protoplasm visible to the naked eye. 


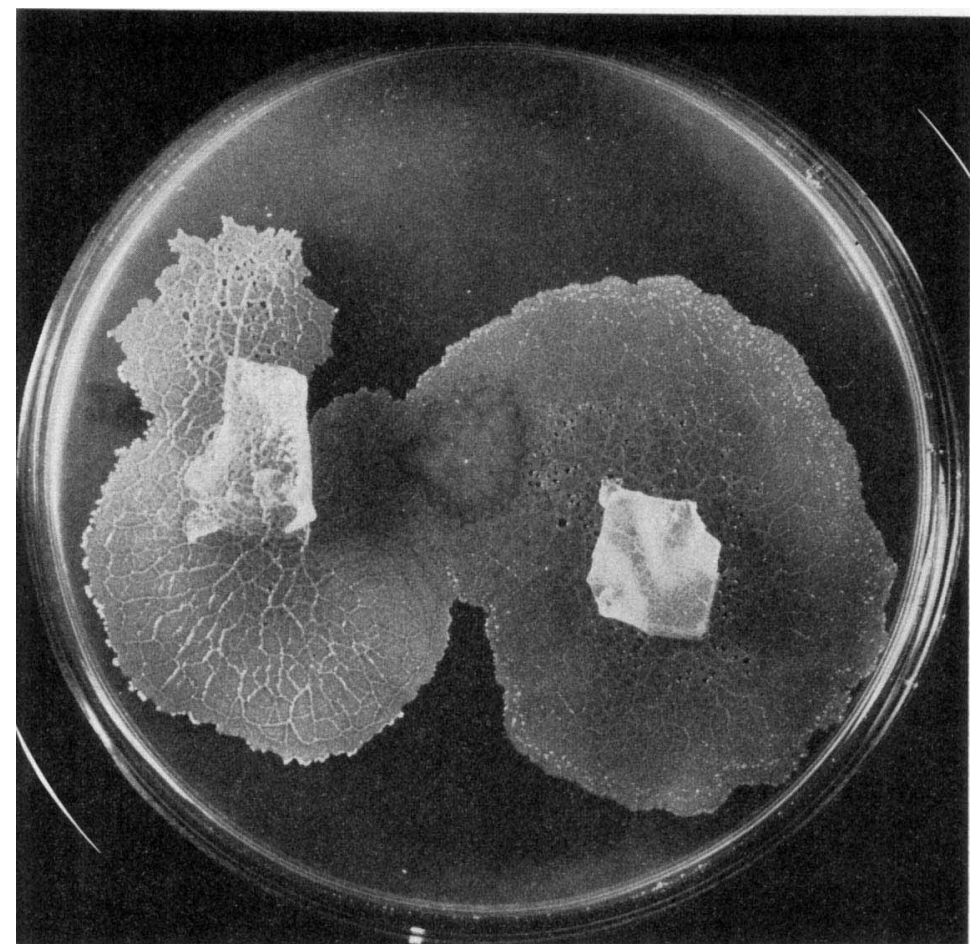

Fig. 2. About 5 min after Fig. I. Further dead areas have appeared and coalescence of existing areas through spreading has occurred.

RESULTS

The normal course of the lethal reaction

When two pieces of agar, bearing plasmodia of strains 15 and 29 respectively were placed about $4 \mathrm{~cm}$ apart on agar medium in a Petri dish, the resulting plasmodia came into contact in $\mathrm{I}$ to 2 days, when both plasmodia were about $2 \mathrm{~cm}$ in radius and then fused with each other in a few minutes. Prominent 'veins' developed between the plasmodia and rapid transport of protoplasm occurred along these channels. A thorough mixing of the protoplasm of the two strains over a wide area was inferred from studies of plasmodial fusion by other workers (Miller, Anderson \& Peterjohn, 1964; Rusch, Sachsenmaier, Behrens \& Gruter, I966; Kerr \& Waxlax, 1968). For about $4 \mathrm{~h}$ the fused plasmodia did not show any abnormalities; up to this point fusion proceeded and a common system of veins was established, as also occurred when plasmodia of the same strain met. About 4 to $5 \mathrm{~h}$ after the onset of plasmodial fusion many of the larger veins became occluded but streaming continued at a reduced rate in smaller veins which formed crossing the occluded veins. The first indication of the lethal reaction visible to the naked eye was the appearance, about $5 \mathrm{~h}$ after fusion, of circular dead areas about I $\mathrm{mm}$ in diameter. These areas increased in size and coalesced and at the same time more such areas appeared (Fig. I, 2). Within about $30 \mathrm{~min}$ of the onset of the reaction a single large dead area was established, increasing in radius at about $\mathrm{I} \mathrm{cm} / \mathrm{h}$ (Fig. 3, 4). Examination of a dead area with the microscope showed that as long as the area continued to spread, its margin was sharply defined although there was no actual physical delimitation. The living plasmodium was granular and showed protoplasmic streaming as 


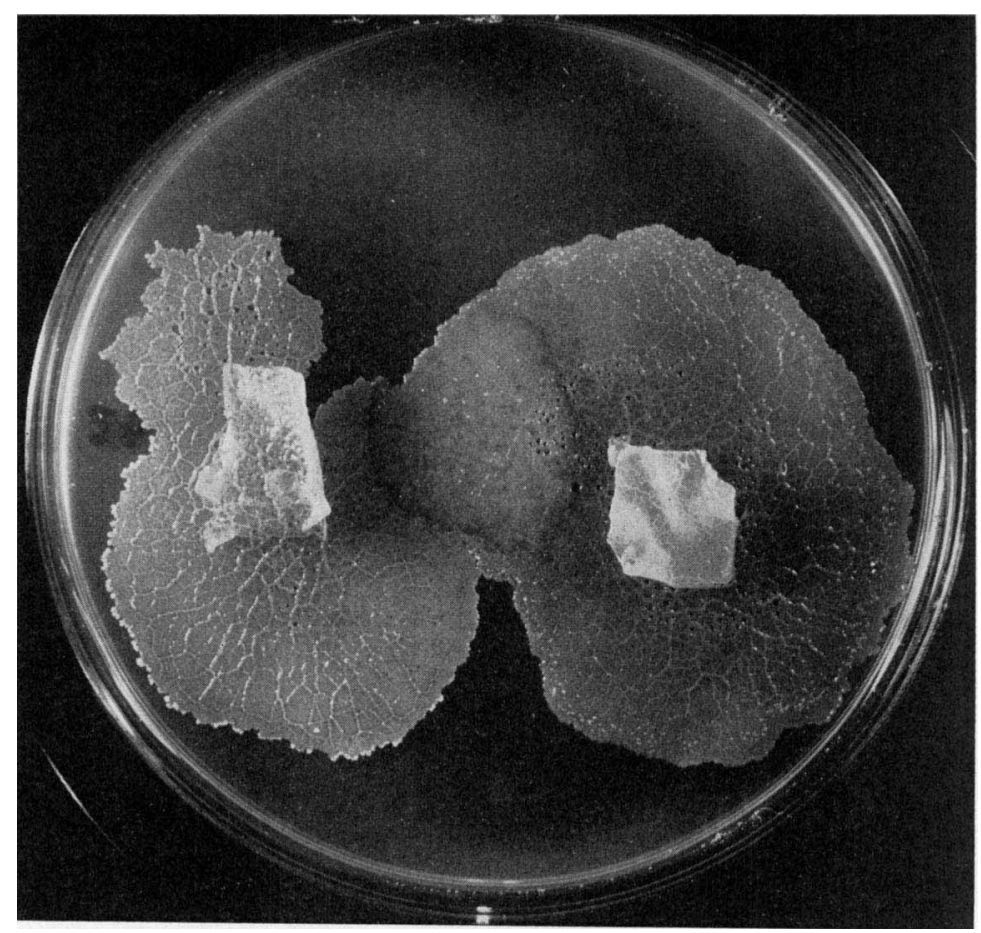

Fig. 3. About 20 min after Fig. I. Further spreading of the dead area has occurred.

well as random movements of granules but close to the dead area streaming ceased and little movement could be seen. At the actual advancing margin of a dead area a brief movement of protoplasm and granules a short distance into the dead area occurred. Most of the granules then disappeared, the smallest in about $30 \mathrm{~s}$ and the largest within $2 \mathrm{~min}$, leaving a clear dead protoplasm with few granules. Viewed by reflected light dead areas were matt, whereas the living plasmodium was glistening. Dead areas intially had a greater transparency, and under the microscope appeared brighter than living areas, but later darkened and decreased in transparency, becoming greyish instead of pale yellow. The surface of dead areas was smooth, whereas that of living plasmodium was irregular. The living plasmodium was soft and could be readily removed from the agar by gentle scraping with a platinum loop, although it was killed in the process. The dead plasmodium could not be removed in this way and with more vigourous scraping cracked and broke into pieces or folded over, and often was sufficiently tough to be pulled away from the agar with forceps.

The spreading of the dead area into the region occupied before plasmodial fusion by strain 15 generally soon ceased. The surviving plasmodium acquired a membrane separating it from the dead material and withdrew leaving a zone a few $\mathrm{mm}$ wide between itself and the dead material. The retreating plasmodium left a great deal of debris in this zone, including irregular particles about the size of nuclei. At this stage the living plasmodium had numerous vacuoles also containing such particles. Food vacuoles were common when plasmodia were grown on particulate substrates such as oatflakes but were normally rare in plasmodia on semidefined medium. Subculture from surviving plasmodium in the region previously occupied by strain 15 yielded plasmodia having the morphology of 15 and able to fuse harmlessly with $I 5$ and lethally with 29 . Finally, the surviving plasmodium reinvaded the 


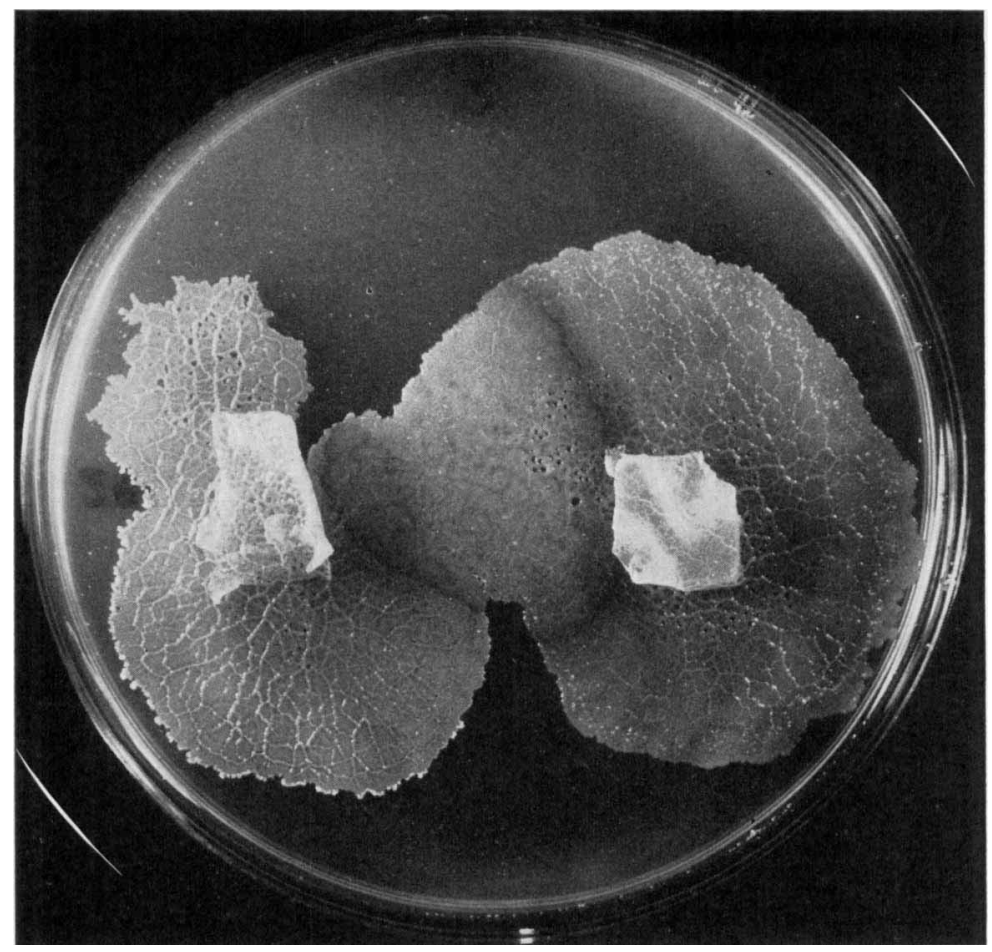

Fig. 4. About $35 \mathrm{~min}$ after Fig. I. The dead area continues to spread rapidly.

area occupied by dead material (Fig. 5,6). It was not adversely affected by contact with the dead material indicating that stable toxic substances were not involved in the lethal reaction. The dead material finally disappeared, digested by the living plasmodium. The spread of the dead area into the region formerly occupied by strain 29 often ceased only when the latter area of plasmodium was wholly destroyed (Fig. 5). If the plasmodium did succeed in delimiting itself from the dead material, a further reaction often occurred, destroying much of the surviving plasmodium. Recovery of viable plasmodium from this region was difficult, hence the lethal reaction normally resulted in the destruction of 29 and its replacement by 15 .

As a lethal reaction advanced along the edge of a plasmodium the plasmodial margin ceased to be sharply defined and an oily film spread on to the agar. Oil droplets were not conspicuous in living plasmodia on semidefined medium but large refractile droplets were common in dead areas resulting from lethal interaction. These observations, suggesting destruction of the plasmodial surface membrane, led to experiments with lysolecithin, a phospholipid capable of disrupting cell membranes (Robinson, I96I). Particles of lysolecithin (Grade II, prepared from egg lecithin, Sigma Chemical Company) were placed on plasmodia. In 5 min most of the material had passed into solution and produced circular dead areas which increased in size for a few minutes until a diameter of about $2 \mathrm{~mm}$ was reached. The appearance of the dead areas and the effects observed at their advancing edges were similar to those produced by lethal interaction and were unlike those resulting from other toxic substances. The decisive phase in lethal interaction, after which recovery was impossible, may therefore have been the disruption of the surface membrane of the plasmodium. Williams \& Wilson (1966) have suggested that death after fusion of incompatible strains of Neurospora crassa results from damage to the cell membrane. 


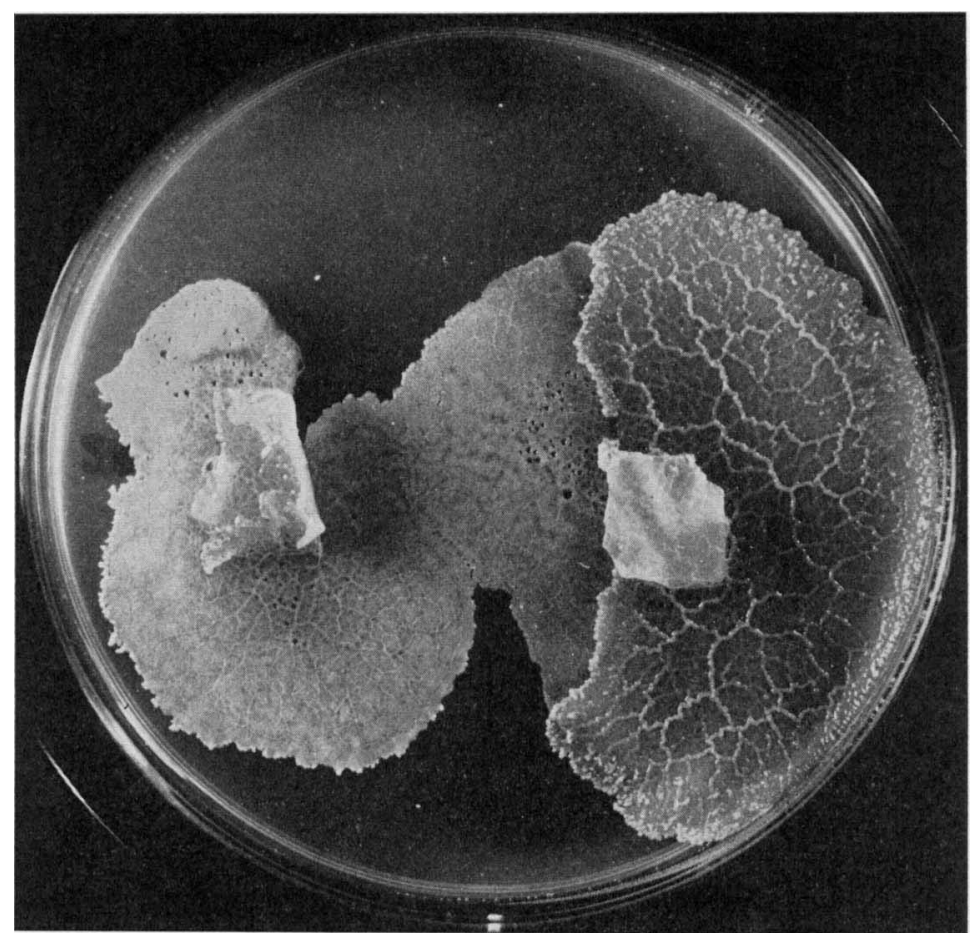

Fig. 5. About $17 \mathrm{~h}$ after Fig. I. The plasmodium on the left is dead and the surviving plasmodium has now become delimited from the dead region.

\section{The influence of nutrition on the lethal reaction}

The effect on the lethal reaction of diluting the medium or reducing the concentration of glucose, peptone and other constituents was investigated (Table I). Lowered peptone levels decreased the frequency and extent of lethal reactions and on plain agar lethal reactions were not observed.

\section{Fusion of plasmodia strains 15 and 29 in various ratios}

Plasmodia of strains 15 and 29 were allowed to spread on medium in which glucose had been replaced by starch; this gives faster spread than the usual medium. Squares of plasmodium of the two strains with underlying agar were cut and placed together in Petri dishes. Fusion between the plasmodia occurred in about $\mathrm{I} h$. If the plasmodia were of equal area a lethal reaction occurred after about $6 \mathrm{~h}$, destroying the entire region previously occupied by 29 and about half of that occupied by 15 (Table 2), a result similar to that obtained when plasmodia of approximately equal size met after growth on semidefined agar medium in Petri dishes (Fig. 5). Fusion between a small area of 15 and a large area of 29 commonly resulted in the destruction of almost the entire plasmodium (Table 2), and lethal reactions have been obtained when the area of 15 was only $4 \%$ of that of 29 . When a small area of 29 fused with a large area of 15 , however, either no reaction was observed or destruction was limited to the region occupied initially by 29 (Table 2 ). 


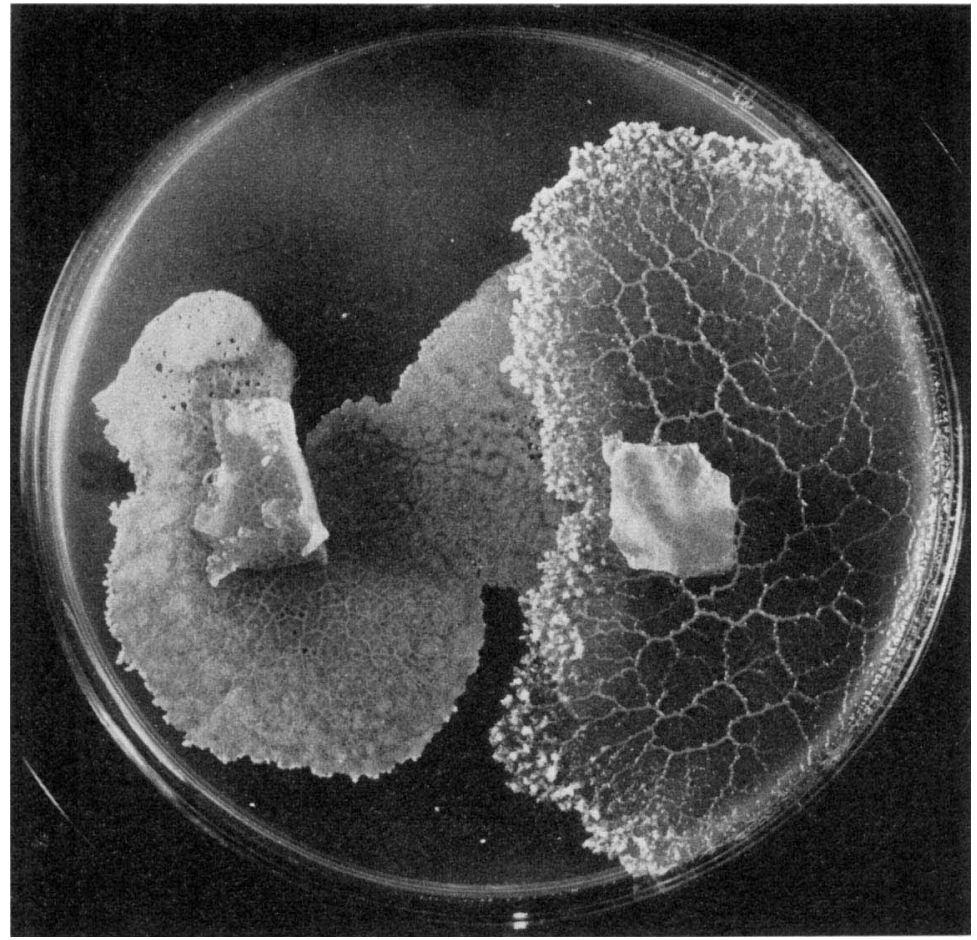

Fig. 6. About $23 \mathrm{~h}$ after Fig. 1. The surviving plasmodium is advancing over agar and dead plasmodium.

Table I. Effect of lowered levels of components of semidefined agar medium on the frequency of the lethal interaction between plasmodia of Physarum polycephalum*

\begin{tabular}{|c|c|c|c|c|c|}
\hline & & entrat & in se & $\mathrm{d} \mathrm{m}$ & \\
\hline & $\mathrm{I} \cdot 0 \dagger$ & 0.5 & 0.2 & O.I & 0 \\
\hline Diluted medium & - & 10 & 9 & 2 & of \\
\hline Lowered glucose & 10 & 10 & IO & IO & $2 \S$ \\
\hline Lowered peptone & IO & 9 & 5 & 3 & -1 \\
\hline Other components lowered & I0 & Io & 10 & 7 & 7 \\
\hline
\end{tabular}

* The number of Petri dishes in which lethal reactions occurred is recorded. Ten Petri dishes in each test, and unless otherwise stated, fusion between strains in all dishes.

$\dagger$ Full strength semidefined medium.

$\$$ Plain agar.

$\S$ In a further five Petri dishes plasmodia separated after fusion leaving microscopic traces of dead material.

\| Fusion did not occur in most Petri dishes, a consequence of death or cessation of growth of one or both plasmodia. Dead areas resulting from peptone deficiency differed from those caused by the lethal reaction in being like butter in appearance and consistency, almost opaque, and readily scraped from the agar with a platinum loop.

\section{Fusion of plasmodia on plain agar}

Ten Petri dishes of plain agar were inoculated with squares (area $\mathrm{I} \cdot 25 \mathrm{~cm}^{2}$ ) of 15 and 29 placed about $4 \mathrm{~cm}$ apart. Rapid migration of plasmodia of both strains occurred and after 2 days fusion between strains had taken place on nine Petri dishes but lethal reactions were not observed. Subcultures were then made on to starch agar. All nine subcultures grew well, had the morphological features of strain 15 and on testing behaved as strain 15 . It is 
Table 2. Effect on the extent of the lethal interaction between plasmodia of Physarum polycephalum of fusing the two strains in various ratios

\begin{tabular}{cccc} 
Strain 15 & \multicolumn{2}{c}{ Areas destroyed $\left(\mathrm{cm}^{2}\right)$} \\
I & Strain 29 & $0 \cdot 32$ & Strain 29 \\
I & 9 & Strain 15 & $7 \cdot$ I I \\
I & 9 & I.00 & $9 \cdot 00$ \\
I & 9 & $0 \cdot 97$ & $9 \cdot 00$ \\
I & 9 & $1 \cdot 00$ & $9 \cdot 00$ \\
5 & 9 & $2 \cdot 52$ & $9 \cdot 00$ \\
5 & 5 & $2 \cdot 69$ & $5 \cdot 00$ \\
5 & 5 & $0 \cdot 07$ & $5 \cdot 00$ \\
5 & 5 & I.53 & $5 \cdot 00$ \\
5 & 5 & $2 \cdot 20$ & $5 \cdot 00$ \\
9 & 5 & 0 & $5 \cdot 00$ \\
9 & I & 0 & 0 \\
9 & I & 0 & $0 \cdot 52$ \\
9 & I & 0 & 0 \\
9 & I & 0 & 0.98 \\
& I & & $0 \cdot 73$
\end{tabular}

concluded that disappearance of strain 29 had occurred in the absence of a lethal reaction, either through the actual elimination of nuclei of strain 29 or through nuclei of strain 15 multiplying more rapidly than those of 29 after transfer of plasmodia to starch agar. Elimination of nuclei of 29 while the plasmodium was on plain agar seems most likely, as the transfer of plasmodia containing the nuclei of both strains to a nutrient medium would be expected to lead to a lethal reaction (see below).

\section{Fusion of microplasmodia of strains 15 and 29}

Culture liquid with suspended microplasmodia was taken from vigorously growing shaken cultures of 15 and 29 at about the same stage of growth, mixed in the proportion I:9 (i.e. strain 15 constituting one-tenth of the mixture) and spread on starch agar in ten Petri dishes. Fusion of microplasmodia occurred during the following 2 days and death of some microplasmodia after fusion was observed. Subcultures (inoculum $\mathrm{I} \cdot 0 \mathrm{~cm}^{2}$ of agar bearing plasmodium) were made after 2 and 7 days from each Petri dish on to starch agar. One subculture made at 2 days grew rapidly and on testing behaved as strain 15 . The other nine subcultures made at 2 days responded to fresh medium in a variety of ways indicating that both genotypes were present in the inoculum. Some spread for about a day, then ceased growth for a few days while debris (in some cultures apparently mainly nuclei and in others dead protoplasm) was discharged, and finally grew rapidly and normally. Others withdrew towards the inoculum block and underwent lethal reactions so extensive that living material was difficult to detect, and some underwent severe lethal reactions without having left the inoculum block. Ultimately, sometimes after a week or to days, rapid growth occurred from traces of surviving plasmodium and all cultures on testing behaved as strain 15, hence it would appear that strain 29 was eliminated either by discharge of the nuclei or through a lethal reaction. Every gradation from the elimination of particles resembling nuclei to extensive lethal reactions visible to the naked eye was observed. All nine subcultures made at 7 days grew normally and behaved as strain 15 . It was concluded that when microplasmodia of 15 and 29 were fused in the ratio $I: 9$, elimination of 29 was complete in 7 days but not in 2, and that transfer of plasmodia in which both types of nuclei were present to fresh media led to a lethal reaction. 


\section{DISCUSSION}

The production of an unstable heterokaryon in Physarum polycephalum through plasmodial fusion has been demonstrated by Poulter \& Dee (I968) who found that fusion of plasmodia of genotype $f_{3} f_{3}$ and $f_{4} f_{4}$ gave a plasmodium behaving initially as $f_{3} f_{4}$ but subsequently reverting to parental type. Kerr (1965) showed that fusion between plasmodia of strains A 20 and C6 of another Myxomycete, Didymium nigripes, was followed by the elimination of strain A 20 as indicated by the gradual and parallel disappearance of two characteristics of A 20. Clark \& Collins (1968) showed the disappearance of a mating-type marker in Didymium iridis heterokaryons and attributed this to nuclear selection against one parental type.

Strain 29 disappears after fusion with strain 15 , either through a lethal reaction or without any such striking manifestation of incompatibility. The disappearance of 29 cannot be attributed to karyogamy between nuclei of 15 and 29 followed by dominance of the more obvious characteristics of 15 , as karyogamy in Physarum polycephalum occurs at plasmodium initiation and plasmodia are diploid. The most reasonable interpretation is therefore nuclear selection against 29 , and its disappearance in the absence of appreciable growth (i.e. after fusion on plain agar) indicates that this selection takes the form of actual elimination of the nuclei of 29 rather than more rapid multiplication of nuclei of 75 . It is suggested that elimination consists of the recognition of alien nuclei, their enclosure in autophagic vesicles, partial digestion and excretion. Ultrastructural studies now in progress (D. J. Border \& M. J. Carlile, to be published) support this hypothesis.

The prominent lethal interaction between strains $I 5$ and 29 occurs when abundant nutrients are available and when the contribution of $I 5$ to the heterokaryon is comparable to or less than that of 29. A range of reactions of intermediate severity between apparent elimination of nuclei and extensive lethal interaction has been observed. It is therefore best to regard the lethal reaction as an extreme expression of the process responsible for the rejection of alien nuclei rather than as a separate phenomenon. Phagocytic reactions against alien particles are known to involve the release of lytic enzymes from lysosomes; it is therefore possible that particularly abundant and active alien nuclei and their products bring about a catastrophically violent reaction.

I thank Dr Jennifer Dee for providing strains of Physarum polycephalum, Miss Jean Carpenter for skilled technical assistance, Mr C. E. Groome for photography and Professor Sir Ernst Chain, F.R.S., for helpful discussion.

\section{REFERENCES}

Carlile, M. J. (1971). Myxomycetes and other slime moulds. In Methods in Microbiology, vol. IV, pp. 237265. Edited by C. Booth. London: Academic Press.

Carlile, M. J. \& Dee, J. (I967). Plasmodial fusion and lethal interaction between strains in a Myxomycete. Nature, London 2r5, 832-834.

Caten, C. E. \& Jinks, J. L. (I966). Heterokaryosis: its significance in wild homothallic Ascomycetes and Fungi Imperfecti. Transactions of the British Mycological Society 49, 8I-93.

Clark, J. \& Collins, O. R. (1968). Diploid mating type heterokaryons and nuclear selection in Didymium iridis. American Journal of Botany 55, 719.

Collins, O. R. \& Haskins, E. F. (1970). Evidence for polygenic control of plasmodial fusion in Physarum polycephalum. Nature, London 226, 279-280.

DANiels, E. W. (1962). Limits of transplantation tolerance in large amoebae. I. Microfusion studies using Amoeba proteus, Pelomyxa illinoisensis, and three strains of Pelomyxa carolinensis. Journal of Protozoology 9, 183-187. 
Fincham, J. R. S. \& Day, P. R. (197I). Fungal Genetics, 3rd edn. Oxford: Blackwell.

FlentJE, N. T. \& Stretron, H. M. (1964). Mechanisms of variation in Thanatephorus cucumeris and T. praticolus. Australian Journal of Biological Science 17, 686-704.

Harris, H. (1970). Cell Fusion. Oxford: Clarendon Press.

JEON, K. W. \& LORCH, I. J. (1969). Lethal effect of heterologous nuclei in amoeba heterokaryons. Experimental Cell Research 56, 233-238.

JeON, K. W., LORCH, I. J. \& DanieLLI, J. F. (1970). Reassembly of living cells from dissociated components. Science, New York 167, 1626-1627.

KERR, N. S. (1965). Disappearance of a genetic marker from a cytoplasmic hybrid plasmodium of a true slime mould. Science, New York 147, I 586-1 588.

KeRR, N. S. \& WaxLAX, J. N. (1968). The fusion of plasmodia of a true slime mould grown on various nutrients and mixing of fused plasmodia. Transactions of the American Microscopical Society 87, 197200.

Miller, D. M., Anderson, J. D. \& Peterjohn, G. W. (1964). Protoplasmic distribution and plasmodial fusion in a myxomycete Physarum polycephalum. Transactions of the Illinois State Academy of Sciences 57, 94-97.

PoulteR, R. T. M. \& DeE, J. (I968). Segregation of factors controlling fusion between plasmodia of the true slime mould Physarum polycephalum. Genetical Research 12, 7I-79.

Power, J. B., Cummings, S. E. \& CockING, E. C. (1970). Fusion of isolated plant protoplasts. Nature, London 225, $1016-1018$.

RichteR, G. (1962). Nuclear-Cytoplasmic Interactions. In Physiology and Biochemistry of Algae, pp. 633-652. Edited by R. A. Lewin. New York: Academic Press.

Robinson, N. (1961). Lysolecithin. Journal of Pharmacy and Pharmacology 13, 321-354.

Rusch, H. P., SACHSENMAier, W., Behrens, K. \& Gruter, V. (I966). Synchronization of mitosis by the fusion of plasmodia of Physarum polycephalum. Journal of Cell Biology 3r, 204-209.

WheAls, A. E. (1970). A homothallic strain of the Myxomycete Physarum polycephalum. Genetics 66, 623633.

Williams, C. A. \& Wilson, J. F. (1966). Cytoplasmic incompatibility reactions in Neurospora crassa. Annals of the New York Academy of Sciences 129, 853-863. 